\title{
Simplex Method for Solving Maximum Problems in Linear Programming
}

\author{
Khin Kye Mon \\ Lecturer \\ Department of Engineering Mathematics \\ Technological University (Mawlamyine) \\ Mawlamyine, Myanmar
}

\begin{abstract}
In this paper, the simplex method in linear programming is discussed for solving maximum problems with constraints. The simplex method is a general mathematical solution technique for solving linear programming problems. In the simplex method, the model is put into the form of a table, and then a number of mathematical steps are performed on the table. This simplex method is an algebraic procedure in which a series of repetitive operations are used to reach the optimal solution.
\end{abstract}

Keywords: simplex method; liner programming; objective function; optimal solution; algebraic procedure

\section{INTRODUCTION}

Optimization principles are of basic importance in modern Engineering design and systems operation in various areas. Optimization problems have both constrained optimization (Gradient method) and unconstrained optimization (linear programming). Graphical method and simplex method are two methods for solving Linear programming problems. For linear programming problems involving two variables, the graphical solution method is convenient. However, for problems involving more than two variables or problems involving a large number of constraints, it is better to use solution methods that are adaptable to computers. One such method is called the simplex method. A linear program is a method of achieving the best outcome given a maximum or a minimum equation with linear constraints. To solve linear programming problems in three or more variables, we can use the simplex method.

\section{METHOD}

The simplex method can be applied for solving the maximum problem. The necessary steps are explained in the followings.

Step (1): Set up simplex tableau using slack variables.

Step (2): Locate pivot value

(i) Look for negative indicator in first row.

(ii) For the value in this column, divide the far right column by each value to find a "test ratio".

(iii) The value with the smallest non negative "test ratio" is pivot.

Step (3): Pivot to find a new tableau.

Step (4): Repeat steps $2 \& 3$ if necessary, Goal: no negative indicators in the first row.

Repeat steps $2 \& 3$ until all numbers on the first row are positive.

Step (5): Read the solution.

\section{PROBLEM SOLVING}

A standard maximum problem is discussed in this paper. The following steps are needed to do before solving the problem. (a).z is to be maximized (b). All variables, $x_{1}, x_{2}, x_{3} \ldots, \geq 0$

(c).All constraints are "less than or equal to (i.e. $\leq$ )

Maximize, $\quad \mathrm{z}=40 \mathrm{x}_{1}+88 \mathrm{x}_{2}$

subject to the constraints, $2 x_{1}+8 x_{2} \leq 60$

$$
5 x_{1}+2 x_{2} \leq 60
$$$$
\mathrm{x}_{1} \geq 0, \mathrm{x}_{2} \geq 0
$$

Next, the first two inequalities are converted to linear equations by introducing two slack variables. Together with the objective function, written as an equation,

$\mathrm{z}-40 \mathrm{x} 1-88 \mathrm{x} 2=0$.

The normal form is

$\mathrm{z}-40 \mathrm{x}_{1}-88 \mathrm{x}_{2}=0$

$2 \mathrm{x}_{1}+8 \mathrm{x}_{2}+\mathrm{x}_{3}=60$

$5 \mathrm{x}_{1}+2 \mathrm{x}_{2}+\mathrm{x}_{4}=60$

$\mathrm{x}_{1} \geq 0, \mathrm{x}_{2} \geq 0, \mathrm{x}_{3} \geq 0, \mathrm{x}_{4} \geq 0$.

\subsection{Simplex Tableau}

To find an optimal solution of it, its augmented matrix must be considered. This is called the "simplex tableau".

$\mathrm{T}_{0}=\left[\begin{array}{ccrccc}\mathrm{z} & \mathrm{x}_{1} & \mathrm{x}_{2} & \mathrm{x}_{3} & \mathrm{x}_{4} & \mathrm{~b} \\ 1 & -40 & -88 & 0 & 0 & 0 \\ 0 & 2 & 8 & 1 & 0 & 60 \\ 0 & 5 & 2 & 0 & 1 & 60\end{array}\right]$

\subsection{Selection of Pivot}

Most negative indicator is found in first row, and then the value in this column divide the far right column of each value to find a test ratio. The value with the smallest non negative "test ratio" is pivot. So, the values are shown in below.

$$
\frac{60}{2}=30, \frac{60}{5}=12
$$

Among them, the smallest value is 12 . Thus, pivot is 5 . 


\subsection{Elimination by Row Operation}

Matrix is altered by using some restricted row operations. One of the entries in the tableau is used as a pivot. The aim is to make all of other elements in the column with the pivot equal to zero. The elements in a row are multiplied by a nonzero constant and added a multiple of one row to the elements of a multiple of any other row.

$$
T_{2}=\left[\begin{array}{cccccc}
\mathrm{z} & \mathrm{x}_{1} & \mathrm{x}_{2} & \mathrm{x}_{3} & \mathrm{x}_{4} & \mathrm{~b} \\
0 & 0 & -72 & 0 & 8 & 480 \\
0 & 0 & 7.2 & 1 & -0.4 & 36 \\
0 & 5 & 2 & 0 & 1 & 60
\end{array}\right] \mathrm{R}_{1}+8 \mathrm{R}_{3} \text { and } \mathrm{R}_{2}-0.4 \mathrm{R}_{3}
$$

When a simplex tableau is considered, it may be able to spot basic variables. A basic variable is a variable that only has all zeros expect one number in its column in the tableau. One of basic feasible solution can be found by finding the value of any basic variables and then setting all remaining variables equal to zero.

Unfortunately, solutions read off of the initial simplex tableau are seldom optimal.

It can be seen that basic variables are now $\mathrm{x}_{1}, \mathrm{x}_{2}$ and nonbasic variables are $x_{3}, x_{4}$. The basic feasible solution is given by $\mathrm{T}_{1}$

$x_{1}=\frac{60}{5}=12, \quad x_{2}=0, \quad x_{3}=\frac{36}{1}=36, \quad x_{4}=0, \quad z=480$.

Elimination is applied only to get non negative entries in row one but the other rows aren't needed to eliminate. So, the basic feasible solution given by $T_{1}$ is not yet optimal because the non-negative entry -72 in row 1 .

Accordingly, the operations are performed again to choose pivot in the column of -72 . The test ratios

$$
\frac{36}{7.2}=5, \frac{60}{2}=30 \text { are got. Select } 7.2 \text { as the pivot because it }
$$

gave the smallest quotient $\frac{36}{7.2}=5$.

By elimination of row operation gives,

$$
\mathrm{T}_{2}=\left[\begin{array}{cccrcc}
\mathrm{z} & \mathrm{x}_{1} & \mathrm{x}_{2} & \mathrm{x}_{3} & \mathrm{x}_{4} & \mathrm{~b} \\
1 & 0 & 0 & 10 & 4 & 840 \\
0 & 0 & 7.2 & 1 & -0.4 & 36 \\
0 & 5 & 0 & \frac{-1}{3.6} & \frac{1}{0.9} & 50
\end{array}\right] \mathrm{R}_{1}+10 \mathrm{R}_{2} \text { and } \mathrm{R}_{3}-\frac{2}{7.2} \mathrm{R}_{2}
$$

It can be seen that basic variables are now $\mathrm{x}_{1}, \mathrm{x}_{3}$ and nonbasic variables are $\mathrm{x}_{2}, \mathrm{x}_{4}$. The basic feasible solution is given by $\mathrm{T}_{2}$

$$
x_{1}=\frac{50}{5}=10, \quad x_{2}=\frac{36}{7.2}=5 \quad x_{3}=0, \quad x_{4}=0, \quad z=840 .
$$

\section{RESULT}

The optimum solution is found out from the above calculation. This is $z=f(10,5)=40 \times 10+88 \times 5=840$. Since $\mathrm{T}_{2}$ contains no more non negative entries in row 1 , this is maximum possible value. This is the solution of our maximum problem by using the simplex method.

\section{CONCLUSION}

In this study, simplex method is applied to solve the maximum problem. The simplex method is an approach for determining the optimal value of the maximum problems. This method produces an optimal solution to satisfy the given constraints and a maximum value. To use the Simplex method, a given maximum problem needs to be in standard form. By applying the steps in this paper, an optimal solution can be obtained.

\section{REFERENCES}

[1] Christopher Griffin, 2009 Linear Programming. Penn State Math.

[2] Finite Math B, 2010 Linear programming: Simplex method. 